\title{
Emprendimiento universitario: una oportunidad académica para traspasar fronteras
}

\section{University entrepreneurship: an academic opportunity to cross borders}

Irma Abrigo Córdova, Ph.D (c)

Universidad internacional del Ecuador, Ecuador

Autor para correspondencia: irabrigoco@uide.edu.ec,irmabrigocordova2016@gmail.com

Fecha de recepción: 28 de Agosto 2017 - Fecha de aceptación: 15 de Enero de 2018

Resumen: El presente trabajo se realizó en la Facultad de Ciencias Administrativas y Económicas de la Universidad Internacional del Ecuador extensión Loja (UIDE-LOJA) y Facultad de Ciencias Administrativas de la Universidad Nacional Mayor de San Marcos (UNMSM) de Lima-Perú, se trata de describir los proyectos de emprendimiento realizados por estudiantes con el asesoramiento de sus docentes; y como se constituyeron en una oportunidad académica para traspasar fronteras. Los estudiantes de la UIDE-LOJA, con el asesoramiento de sus docentes, realizaron entre los principales, los siguientes proyectos de emprendimiento: Guaviduca green life, Jugo de Granadilla con Miel de Abeja y sus derivados, café molido y mermelada de café, Chicha Lojana enriquecida con Guaviduca. Al respecto, la delegación ecuatoriana viajó al país vecino, a la Feria Binacional "Ideando San Marcos" que tuvo lugar en la UNMSM, en el mes de noviembre de 2016, participando en calidad de "Expositores Invitados" de proyectos de emprendimiento internacional, en las fiestas de la Facultad de Ciencias Administrativas. De igual manera profesores y estudiantes de la UNMSM, también trabajaron proyectos de emprendimiento, entre los más destacados: café "Don Negrito" y sus derivados, Merchandising que identifique tanto al cliente interno como externo con la organización, Piscalicha, Yogurt natural y nutritivo hecho a base de cáscaras de frutas. La representación peruana, partió a Ecuador, con la finalidad de participar en la Casa Abierta de la UIDE-LOJA, desarrollada en el mes de junio de 2017, como "Expositores Invitados" de proyectos de emprendimiento internacional, en las fiestas de la institución universitaria. En los trabajos presentados por las embajadas citadas, se evidenció: investigación, creatividad y trabajo en equipo, así como el uso de las $4 \mathrm{P}$ del emprendimiento o también conocido como marketing mix: precio, producto, promoción y plaza.

Palabras clave: Idea de negocios; producto; precio; plaza; promoción

Abstract: The present work was carried out in the Faculty of Administrative and Economic Sciences of the International University of Ecuador extension Loja (UIDE-LOJA) and Faculty of Administrative Sciences of the Greater National University of San Marcos (UNMSM) of LimaPeru. Describe the entrepreneurship projects carried out by students with the advice of their teachers; And how they became an academic opportunity to cross borders. The students of UIDELOJA, with the advice of their teachers, carried out the following projects: Guaviduca green life, Juice of Granadilla with honey of honey and its derivatives, ground coffee and coffee jam, Chicha Lojana Enriched with Guaviduca. In this regard, the Ecuadorian delegation traveled to the 
neighboring country, to the Binational Fair "Ideando San Marcos" that took place in the UNMSM, in November 2016, participating as "Invited Exhibitors" of international entrepreneurship projects, in The festivities of the Faculty of Administrative Sciences. Likewise, professors and students of the UNMSM also worked on entrepreneurship projects, among the most outstanding ones: "Don Negrito" coffee and its derivatives, Merchandising that identifies both the internal and external customer with the organization, Piscalicha, natural and nutritious yogurt made the base of fruit peels. The Peruvian delegation left for Ecuador to participate in the UIDE-LOJA Open House, held in June 2017, as "Invited Exhibitors" of international entrepreneurship projects, in the festivities of the university institution. Researches, creativity and teamwork, as well as the use of the $4 \mathrm{P}$ of the enterprise or also known as marketing mix: price, product, promotion and place, were evidenced in the works presented by the mentioned embassies.

Key Words: business idea; product; price; square; promotion

\section{Introducción}

\section{Problema o argumento}

Se realizó en la Facultad de Ciencias Administrativas y Económicas de la Universidad Internacional del Ecuador extensión Loja (UIDE-LOJA) y Facultad de Ciencias Administrativas de la Universidad Nacional Mayor de San Marcos (UNMSM) de Lima-Perú, se trata de puntualizar el aporte de docentes y estudiantes, en el desarrollo de proyectos de emprendimiento y como se constituyeron en una oportunidad académica para traspasar fronteras.

\section{Revisión de literatura}

La investigación planteada representa el trabajo académico de proyectos de investigación, desarrollados en la Facultad de Ciencias Administrativas y Económicas de la Universidad Internacional del Ecuador extensión Loja; y Facultad de Ciencias Administrativas de la Universidad Nacional Mayor de San Marcos de Lima-Perú, los cuales, fueron presentados en cada una de las instituciones universitarias, debiéndose trasladar a cada país, con la finalidad de participar en calidad de "Expositores Invitados", una experiencia sin precedentes, tanto para estudiantes como docentes, este acontecimiento, marcó la importancia del intercambio cultural y la confraternidad entre países hermanos. El objetivo de la propuesta es difundir los proyectos de emprendimiento presentados por las instituciones universitarias copartícipes (Alcaraz, 2011; Cohen \& Asín, 2014).

Particularmente, el modelo educativo de la UIDE-LOJA, contempla las estrategias de enseñanza aprendizaje como son "aprender a aprender" "y aprender haciendo", de esta manera se vincula la teoría con la práctica; también se aprecia en la malla curricular la asignatura de "Emprendedores", los factores mencionados, ayudan al estudiante en la adquisición de conocimientos significativos; permitiendo que el educando sea una "persona que identifique una oportunidad de negocio, de un producto o servicio, empiece un proyecto por su propio entusiasmo; posea la capacidad organizativa, sea analítico, entusiasta, innovador, optimista, flexible, dinámico, capaz de asumir riesgos, creativo y orientado al crecimiento" (Prieto, 2014). 
Los proyectos presentaron se iniciaron con una idea de negocio, utilizando en algunos casos, elementos clave a considerar, planteados por (Carvajal, 2010), los cuales se detallan a continuación:

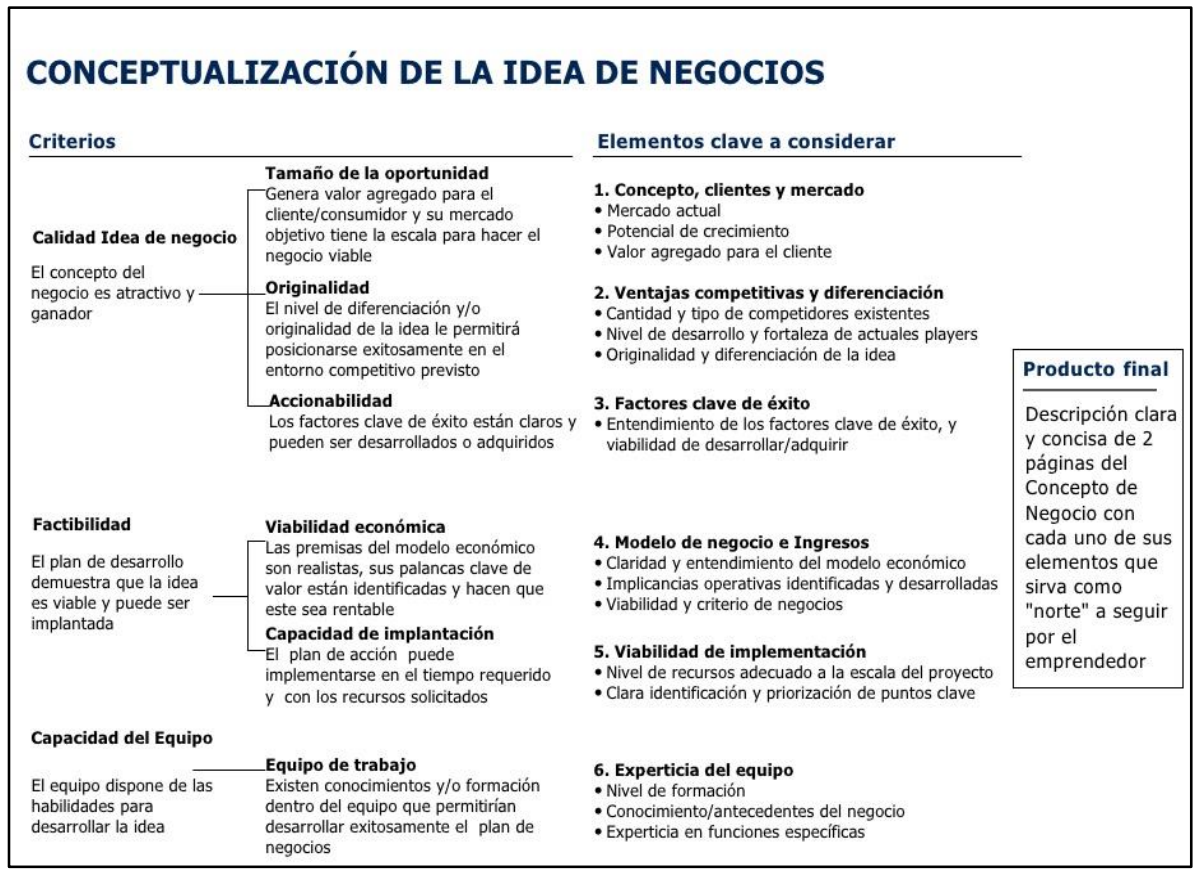

Cuadro $\mathrm{N}^{\circ}$ 01: Conceptualización de la idea de negocios.

Fuente: Datos tomados de Desarrollando una idea de negocios: cómo llegar de una idea a un caso de negocios (2010).

Como todo proyecto de emprendimiento, se observó la aplicación de las $4 \mathrm{p}$ del marketing mix (Rodríguez, Ammetler, \& López, 2006), denominado también, las 4 ps del marketing operativo o táctico (De la Torre, 2016), descritas como sigue:

1. Producto. En los proyectos de emprendimiento peruano, se presentaron las siguientes ideas de negocio:

- Café "don negrito" y sus derivados.

- Merchandising Unico

- Piscalicha.

- SillpiMix.

Los proyectos de emprendimiento ecuatoriano, a continuación, se enlistan:

- Jugo de Granadilla con Miel de Abeja.

- Procafé.

- Chicha Lojana enriquecida con Guaviduca.

- Guaviduca Green Life.

Cada una de ellas cumplió con el proceso de etiquetado, marca y presentación adecuada de los productos. En este acápite, se debe indicar, respecto a la representación ecuatoriana, únicamente viajaron los dos primeros grupos, los restantes no lo pudieron realizar por motivos personales. 
2. Precio. "Es el valor monetario que se otorga a cada producto. Es un factor de mucha importancia en la aceptación del mismo por el target" (De la Torre, 2016). Se estableció el precio de costo y de venta, de cada producto, convirtiéndolo posteriormente a la moneda utilizada en los países participantes, como es el dólar para Ecuador y el sol para Perú.

3. Plaza o distribución. "La distribución engloba todas las actividades que posibilitan el flujo de productos desde la empresa que los fabrica o produce hasta el consumidor final" (Rodríguez, Ammetler, \& López, 2006). En este sentido, cada uno de los emprendedores universitarios llevo a los países participantes sus productos, con la finalidad de ser distribuidos en los eventos organizados por las instituciones mencionadas anteriormente. "Los canales de distribución son el conjunto de personas y empresas comprendidas en la transferencia de derechos de un producto al paso de éste del productor al consumidor final (Stanton, Etzel, \& Walker, 2007). Los productos fueron ofertados, en cada país, logrando la aceptación de la comunidad peruana y ecuatoriana, quienes no dudaron en adquirir mercancías novedosas de los países vecinos (Hitt, Ireland, \& Hoskisson, 2014; Lerma \& Bárcena, 2013)

4. Promoción o comunicación. En los proyectos de emprendimiento se consideró la publicidad, la cual "se define como un segmento operativo dentro de la actividad promocional sustentado por acciones inminentemente comerciales en donde la organización busca hacer ventas y garantizarse un lugar en el mercado", para este fin, los participantes emplearon algunas estrategias como promoción de ventas, merchandising, mercadeo directo, etc. (Thompson, Strickland \& Gamble, 2008; Wheelen \& Hunger, 2013; Prieto, 2014)

\section{Metodología}

Se utilizó la investigación cualitativa, empleando técnicas de recolección de la información como son:

Observación participante: permitió involucrarse en cada grupo de trabajo, visitando cada país participante, con el objetivo de observar las actividades realizadas en cada emprendimiento investigando.

Entrevista semiestructurada: se la aplicó a los docentes y estudiantes de las instituciones universitarias citadas, previamente se ordenaron preguntas para guiar el trabajo, llegando a una amena comunicación con los entrevistados, lo cual permitió conocer los mejores proyectos para ser presentados en las naciones partícipes.

Grupos focales: la aplicación de esta técnica facilitó el trabajo con representantes de la UIDE-LOJA y la UNMSM-LIMA, con quienes se coordinó la intervención de docentes y estudiantes, en calidad de "Asesores y Expositores Invitados", respectivamente. También se analizaron los requerimientos y logística de cada grupo, para la eficiente participación en las instituciones referidas.

Descripción de los participantes: pertenecen a la Facultad de Ciencias Administrativas y Económicas de la Universidad Internacional del Ecuador extensión Loja; y Universidad 
Nacional Mayor San Marcos de Lima-Perú, contando con un número de participación de seis estudiantes y tres docentes de Ecuador; y once estudiantes y tres docentes de Perú.

\section{Resultados}

\section{Proyectos de emprendimiento de la UIDE-LOJA}

La delegación ecuatoriana se presentó en la Feria de emprendimiento "Ideando San Marcos", la cual, fue organizada por la Facultad de Ciencias Administrativas de la UNMSMLIMA; tuvo lugar en las instalaciones de la Universidad Nacional Mayor San Marcos de Lima, en el mes de noviembre del 2016; cabe recalcar, los estudiantes participaron en calidad de "Expositores Invitados", exhibiendo los siguientes proyectos:

\section{Jugo de Granadilla con Miel de Abeja}

Proyecto creado para la elaboración y preparación de jugo de granadilla con miel de abeja, la materia prima se la encuentra disponible en la provincia de Loja; además tanto la fruta como la miel de abeja se la ubican en cualquier época del año. En el entorno lojano, la mayor parte de la población consume granadilla, por ese motivo, el grupo de emprendimiento, utilizó la fruta para realizar el jugo con miel de abeja. A continuación, se aprecia la presentación del producto y exposición de estudiantes.

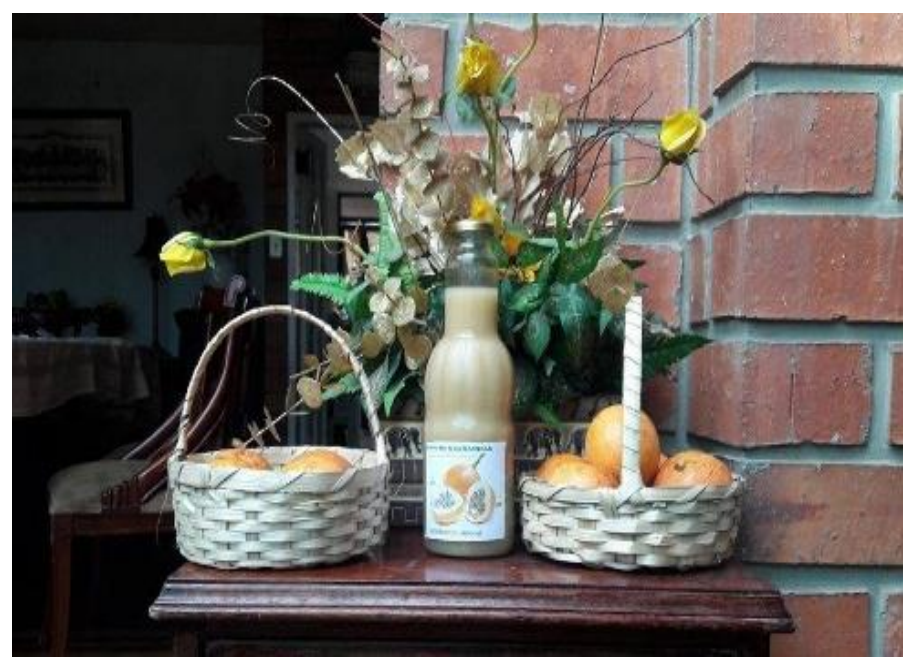

Figura 1. Presentación del producto: Jugo de Granadilla con miel de abeja.

\section{ProCafé}

Proyecto creado para la elaboración y preparación de café tostado y café molido, y con un nuevo producto a basé de frutas, almíbar y café dando como producto final la mermelada de café.

En la preparación del café molido, el grupo de emprendimiento, ofrece una nueva imagen e ideas en lo que es el producto común, en las bolsas de 200G y 400G en base a tela y yute, evitando de esa manera, la utilización de papel para las bolsas del producto. En lo que respecta a la mermelada, se brinda el valor agregado que es el café más el almíbar, con la intención de 
mostrar al público otra manera de consumir el café, este producto fue presentado en envase de vidrio y una tapa dorada con botón. Se observa en los siguientes gráficos, la presentación del producto y exposición de estudiantes.

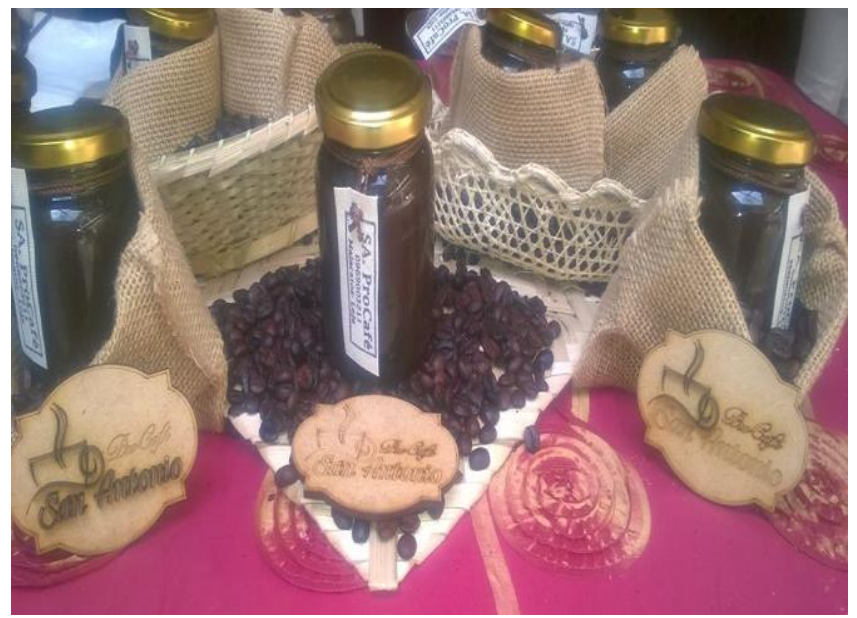

Figura 2. Presentación del producto: Emprendimiento ProCafé.

\section{Proyectos de emprendimiento de la UNMSM-LIMA}

La delegación del país vecino, Perú, exhibió sus productos de emprendimiento en la "Casa Abierta" de la Universidad Internacional del Ecuador extensión Loja, actividad desarrollada en la Plaza de San Sebastián, en el mes de mayo del presente; es importante señalar, igual que en el caso anterior, los estudiantes intervinieron como "Expositores Invitados", presentando los siguientes proyectos, entre los principales:

Yogurt natural y nutritivo hecho a base de cáscaras de frutas "SillpiMix"

El modelo de negocio gira en torno a los productos lácteos, según expresa el equipo de trabajo, el yogurt es el principal producto interesado en lanzar al mercado. La propuesta presenta las siguientes características:

- Insumos de cáscaras de frutas con alto contenido nutricional.

- Producto natural

- Diseño y presentación innovador a un público objetivo poco estudiado (personas que estudian y que trabajan).

Se analiza en los siguientes gráficos, la presentación del producto y exposición de estudiantes. 


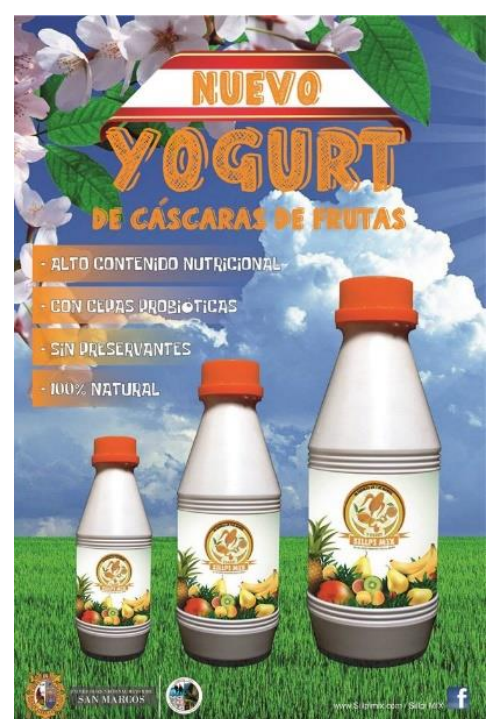

Figura 3. Presentación del producto: Yogurt natural y nutritivo hecho a base de cáscaras de frutas "SillpiMix"

\section{Piscalicha}

La Piscalicha es un aperitivo innovador $100 \%$ orgánico, es una bebida deliciosa, natural, nutritiva y saludable, además de ser energética, digestiva, antioxidante, vitamínica; por sus componentes es preventiva de enfermedades degenerativas, entre otras. Es un producto que impulsa el desarrollo de los componentes de la bebida, siendo esta fuente generadora de recursos para productores de insumos. En los gráficos se evidencia el trabajo desarrollado por el presente grupo.

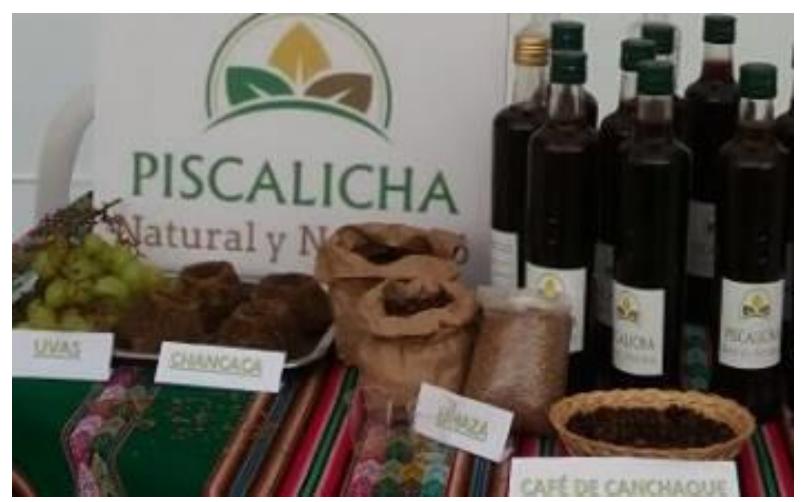

Figura 4. Exposición de Emprendimiento: Piscalicha

\section{Merchandising "Único”}

Este proyecto presenta diferentes productos (Merchandising) de buena calidad con diseños y frases que acrecientan el sentimiento de pertenencia y conocimiento de la cultura de la organización con la cual se trabajará, esto incluye todo tipo de organizaciones (públicas y privadas), los mismos, varían de acuerdo a la naturaleza de la organización, en esta ocasión los productos fueron dirigidos a la comunidad lojana y Universidad Internacional del Ecuador extensión Loja. 


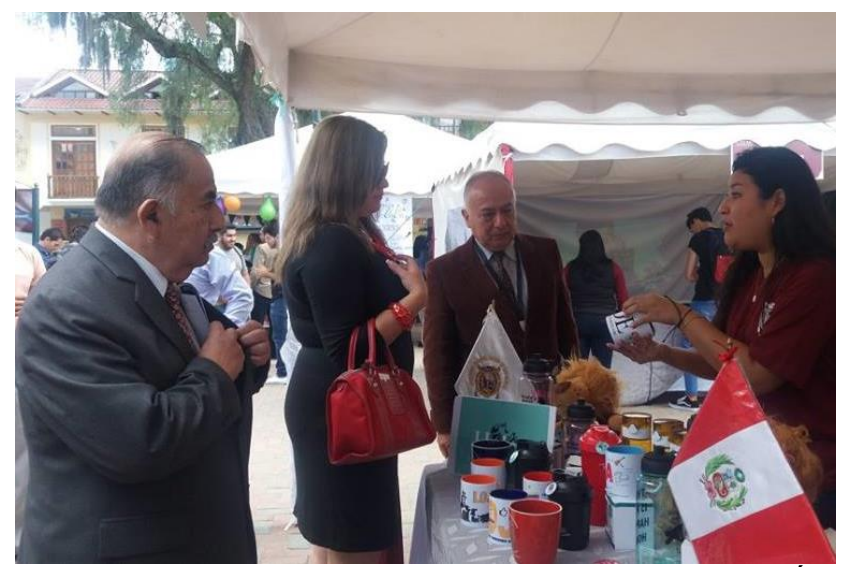

Figura 5. Exposición de Emprendimiento: Merchandising "Único"

\section{Entrega de certificados “Expositores Invitados de proyectos de emprendimiento internacional"}

A continuación, se ubica evidencias de entrega de certificados por la participación de docentes y estudiantes en los eventos académicos llevados a cabo en cada país.

\section{Delegación de la UIDE-LOJA en la UNMSM-LIMA}

Causó gran expectativa la presencia de estudiantes ecuatorianos en la Feria de emprendimiento "Ideando San Marcos", siendo los primeros en llegar a la Universidad Nacional Mayor San Marcos, por invitación del Decano de la Facultad de Ciencias Administrativas, Mgs. Robert Miranda. En el siguiente gráfico se evidencia la entrega de certificados a la delegación ecuatoriana.

\section{Delegación de la UNMSM-LIMA en la UIDE-LOJA}

Igual que el caso anterior, la presencia de estudiantes peruanos en la Casa Abierta, tuvo gran acogida por la comunidad universitaria y lojana, al observar productos novedosos que no existen en la localidad; también los estudiantes San Marquinos, han sido hasta la actualidad, los primeros en llegar a la Universidad Internacional del Ecuador extensión Loja, invitación retribuida por la Directora de la UIDE-LOJA, Mgs. Johanna Vivanco Ochoa, según acuerdo realizado en noviembre 2016. Se aprecia en el gráfico la entrega de certificados a la delegación peruana.

\section{Discusión}

Se empleó la metodología cualitativa, aplicando las técnicas de recolección de la información como son: observación participante, entrevista semiestructurada y grupos focales; este proceso permitió el conocimiento de los diferentes proyectos de emprendimiento que fueron presentados en las instituciones universitarias de Ecuador y Perú. 
Con el empleo de las técnicas citadas se pudo conocer las distintas estrategias de emprendimiento desarrolladas en la UIDE-LOJA y UNMSM-LIMA, logrando identificar similares procesos de enseñanza aprendizaje entre países hermanos.

La experiencia académica se constituyó en una oportunidad para traspasar fronteras a través de proyectos de emprendimiento, evidenciándose la preparación y entrega de cada docente y estudiante para asumir este reto de nivel internacional y convertirse en embajadores de sus países al llevar parte de su cultura.

La propuesta permitió desarrollar una breve descripción de cada uno de los proyectos tanto en Ecuador, como en Perú; además, se realizó una revisión bibliográfica y con la colaboración de proyectos de emprendimiento anteriores desarrollados con estudiantes de UIDELOJA en la asignatura de "Emprendedores", se enfocó la propuesta para presentar un producto terminado que plasme los procesos de emprendimiento llevados a cabo en las instituciones universidades copartícipes (Baca, 2010).

Cabe recalcar, se concluyó la participación de los estudiantes y docentes, de los países citados, con la entrega de Certificados, en reconocimiento a su excelente trabajo, constituyéndose en un ejemplo a seguir; y como pioneros de este tipo de eventos, actualmente la UNMSM-LIMA, está organizando la II Feria de Emprendimiento "Ideando San Marcos", en la cual Ecuador será representada por la UIDE-LOJA, además de contar con delegaciones de: México, Colombia y Argentina.

\section{Conclusión}

La Universidad Internacional del Ecuador extensión Loja, cumple con su modelo pedagógico "Aprender a aprender" y "Aprender haciendo", vinculando la teoría con la práctica, al trabajar con sus estudiantes proyectos de emprendimiento, constituyéndose a la vez en una alternativa para traspasar fronteras; intercambiar ideas de emprendimiento de nivel internacional, cultura y tradiciones, de esta manera, se logrará aprendizajes significativos en los estudiantes de las instituciones universitarias en estudio y se estrecharán los lazos fraternos entre países hermanos.

\section{Bibliografía}

Alcaraz, R. (2011). El emprendedor de éxito. México, D.F.: Editores, S.A. de C.V.

Baca, G. (2010). Evaluación de proyectos. México: Programas Educativos S.A. de C.V.

Carvajal, P. (5 de Febrero de 2010). Desarrollando una idea de negocios: cómo llegar de una idea a un caso de negocios. . Bogotá, Colombia.

Cohen, D., \& Asín, E. (2014). Tecnologías de la Información. México: Mexicana. Núm.736.

De la Torre, L. (2016). Investigación de mercados. Lima: Editora Macro EIRL. 
Díaz, C. (2012). "Impacto de la apropiación de las TIC en la productividad de la empresa manufacturera de Cartagena (Colombia): modelación y creación de mecanismos de adaptación”. 407-408. Cartagena, Colombia.

Gómez, Á., \& Otero, C. (2013). Redes sociales en la empresa. Bogotá: Ediciones de la U.

Hitt, Ireland, \& Hoskisson. (2014). Administración Estratégica. México D.F.: Vacha, S.A. de C.V.

Lerma y Kirchner, A., \& Bárcena, S. (2013). Planeación Comercial. Guía práctica. Colombia: Alfaomega Grupo Editor, S.A. de C.V. México.

Prieto, C. (2014). Emprendimento. Conceptos y plan de negocios. México: Pearson Educación de México, S.A. de C.V.

Rodríguez, I., Ammetler, G., \& López, Ó. (2006). Principios y estrategias de marketing. Barcelona: Editorial UOC.

Stanton, W., Etzel, M., \& Walker, B. (2007). Fundamentos de Marketing. México, D.F.: McGramHill/interamericana Editores S.A. de C.V.

Silva, J. (2013). Emprendedor hacia un emprendimiento sostenible. Colombia: Alfaomega Colombiana S.A.

Thompson, A., Strickland, A., \& Gamble, J. (2008). Administración Estratégica. México D.F.: Editores S.A. de C.V.

Wheelen, T., \& Hunger, D. (2013). Administración Estratégica. Bogotá: Delfín Ltda. 